\title{
Multimodal imaging brain connectivity analysis (MIBCA) toolbox: preliminary application to Alzheimer's disease
}

\author{
Andre Santos Ribeiro ${ }^{1,2^{*}}$, Luís Miguel Lacerda ${ }^{2,3}$, Nuno André da Silva ${ }^{4}$, Hugo Alexandre Ferreira ${ }^{2}$ \\ From PSMR14: 3rd Conference in PET/MR and SPECT/MR \\ Kos Island, Greece. 19-21 May 2014
}

${ }^{1}$ Center for

Neuropsychopharmacology, Division of Brain Sciences, Department of Medicine, Imperial College London, London, UK
The Multimodal Imaging Brain Connectivity Analysis (MIBCA) toolbox is a fully automated all-in-one connectivity analysis toolbox that offers both pre-processing, connectivity, and graph theory analysis of multimodal images such as anatomical, diffusion, and functional MRI, and PET [1]. In this work, the MIBCA functionalities were used to study Alzheimer's Disease in a multimodal MR/PET approach.

Data from 11 healthy subjects and 10 AD patients were obtained from the Alzheimer's Disease Neuroimaging Initiative (ADNI) database (adni.loni.usc.edu), including T1-weighted (T1w), Diffusion Tensor Imaging (DTI) data, and ${ }^{18}$ F-AV-45 (florbetapir) dynamic PET data from 40-60 min post injection (4x5min). Both MR and PET data were automatically pre-processed for all subjects using MIBCA. The T1w data was parcellated into cortical and subcortical regions-of-interest (ROIs), and the corresponding thicknesses and volumes were calculated. DTI data was used to compute structural connectivity matrices based on fibers connecting pairs of ROIs. Lastly, dynamic PET images were summed, and the Standard Uptake Values calculated for each ROI.

An overall higher uptake of ${ }^{18} \mathrm{~F}-\mathrm{AV}-45$, consistent with an increased deposition of amyloid-Beta, was observed for the AD group. Additionally, patients showed significant cortical atrophy (thickness and volume) especially in the enthorhinal and temporal areas, and a significant increase in Mean Diffusivity (MD) in the hippocampus, amygdala and temporal areas, Figure 1. Furthermore, patients showed an overall decrease of both inter- and intra- hemispherical structural connections (tracts), Figure 2. Finally, the 3D-graph visualization showed that the structural loss was global and asymmetric, Figure 3.

This work shows the potential of the MIBCA toolbox for the study of AD, as findings were shown to be in agreement with the literature [2-4]. Here, only structural changes and beta amyloid accumulation were considered. Yet, MIBCA is further able to process fMRI and different radiotracers, and combine all the information in order to provide new insights into AD.

\section{SpringerOpen ${ }^{\circ}$}

(C) 2014 Ribeiro et al; licensee Springer This is an Open Access article distributed under the terms of the Creative Commons Attribution License (http://creativecommons.org/licenses/by/4.0), which permits unrestricted use, distribution, and reproduction in any medium, provided the original work is properly cited. 


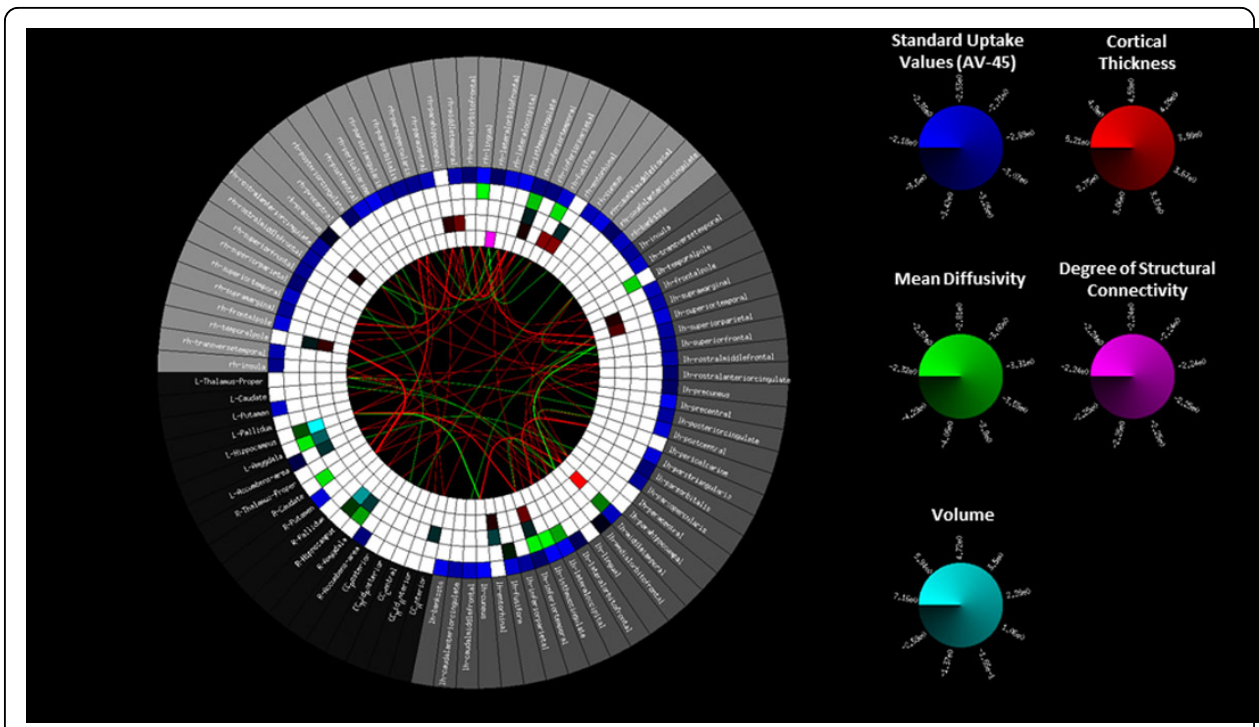

Figure 1 Group difference Connectrogram. Significant differences between Healthy Subjects and AD patients are highlighted in color in the inner rings. From outside to inside rings: region-of-interest (ROI), Standard Uptake Value (18F-AV-45), mean diffusivity, ROI volume, cortical thickness, node degree computed from structural connectivity.

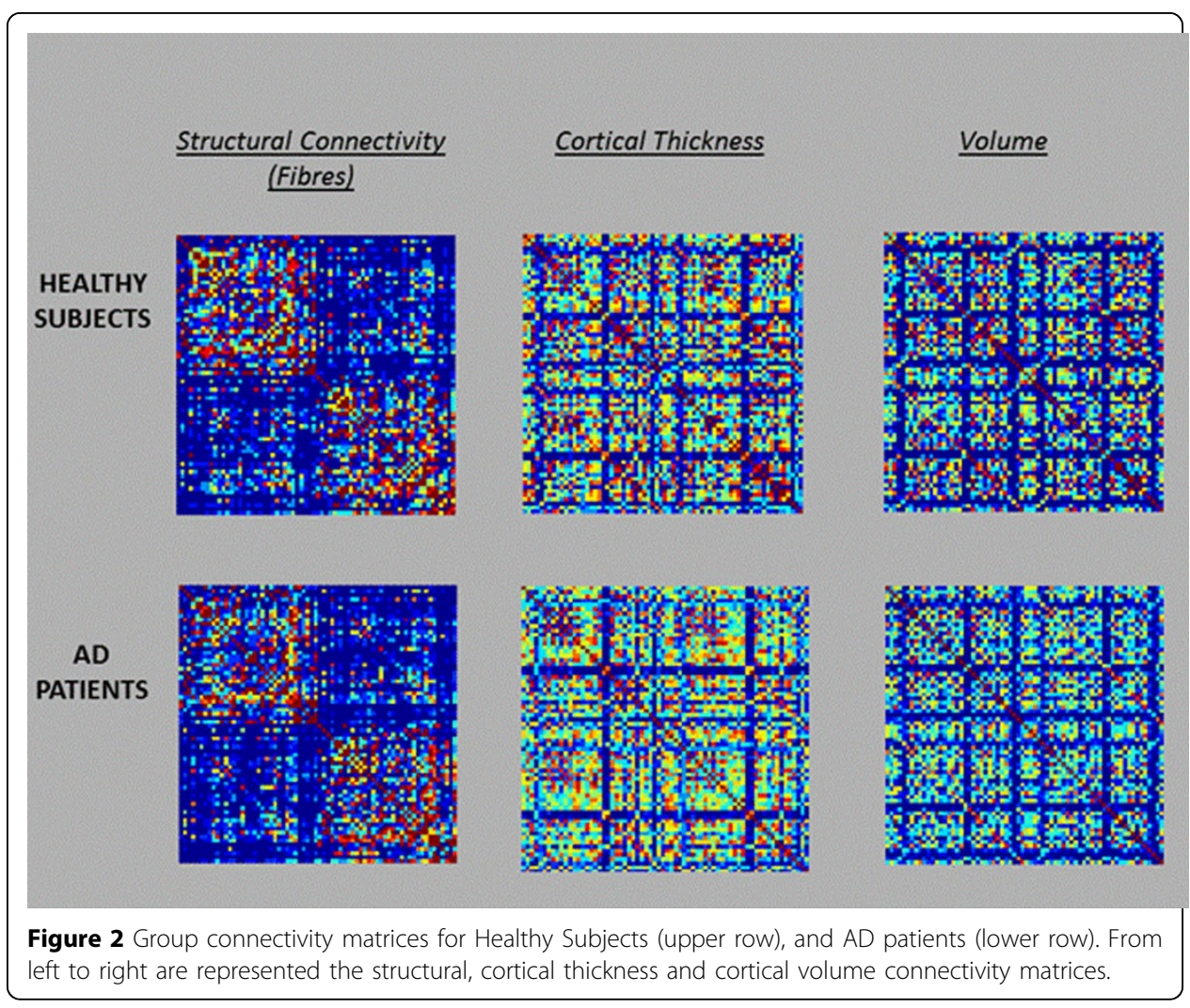

\section{Authors' details}

${ }^{1}$ Center for Neuropsychopharmacology, Division of Brain Sciences, Department of Medicine, Imperial College London, London, UK. ${ }^{2}$ Institute of Biophysics and Biomedical Engineering, Faculty of Sciences of the University of Lisbon, Lisbon, Portugal. ${ }^{3}$ Centre for Neuroimaging Sciences, Institute of Psychiatry, King's College London, London, Denmark Hill, UK. ${ }^{4}$ Institute of Neuroscience and Medicine-4, Forschungszentrum Juelich GmbH, Juelich, Germany. 


\section{References}

1. Santos-Ribeiro A, Lacerda L, Rodrigues J, Sousa JM, Ferreira HA: Multimodal imaging brain connectivity analysis. ESMRMB Congress 30th Annual Scientific Meeting of the European Society for Magnetic Resonance in Medicine and Biology Toulouse, France; 2013

2. Johnson KA, Sperling RA, Gidicsin CM, Carmasin JS, Maye JE, Coleman RE, Reiman EM, Sabbagh MN, Sadowsky CH, Fleisher AS, Murali Doraiswamy P, Carpenter AP, Clark CM, Joshi AD, Lu M, Grundman M, Mintun MA, Pontecorvo MJ, Skovronsky DM: Florbetapir (F18-AV-45) PET to assess amyloid burden in Alzheimer's disease dementia, mild cognitive impairment, and normal aging. Alzheimers Dement 2013, 9:S72-83.

3. Duara R, Loewenstein DA, Potter E, Appel J, Greig MT, Urs R, Shen Q, Raj A, Small B, Barker W, Schofield E, Wu Y, Potter $\mathrm{H}$ : Medial temporal lobe atrophy on MRI scans and the diagnosis of Alzheimer disease. Neurology 2008 71(24):1986-1992.

4. Acosta-Cabronero J, Alley S, Williams GB, Pengas G, Nestor PJ: Diffusion Tensor Metrics as Biomarkers in Alzheimer's Disease. PLOS ONE 2012

doi:10.1186/2197-7364-1-S1-A61

Cite this article as: Ribeiro et al: Multimodal imaging brain connectivity analysis (MIBCA) toolbox: preliminary application to Alzheimer's disease. EJNMMI Physics 2014 1(Suppl 1):A61.

\section{Submit your manuscript to a SpringerOpen ${ }^{\circ}$} journal and benefit from:

- Convenient online submission

Rigorous peer review

- Immediate publication on acceptance

- Open access: articles freely available online

- High visibility within the field

- Retaining the copyright to your article

Submit your next manuscript at $\gg$ springeropen.com 